\title{
Visualisation and quantitative analysis of the near nozzle formation and structure of a high pressure water jet in air and in water
}

\author{
Sarah Jasper*1, Jeanette Hussong ${ }^{2}$, Ralph Lindken ${ }^{1}$ \\ ${ }^{1}$ Institute of Thermodynamics and Fluid Mechanics, Bochum University of Applied Sciences, \\ Lennershofstr. 140, 44801 Bochum \\ ${ }^{2}$ Chair of Hydraulic Fluid Machinery, Ruhr-University Bochum, Universitätsstr. 150, \\ 44801 Bochum \\ ${ }^{*}$ Corresponding author: sarah.jasper@hs-bochum.de
}

\begin{abstract}
High pressure water jets (HPWJ) with Reynolds numbers in the scale of $10^{4}$ are visualised by high speed photography in air and water. Moreover, suitable measurement techniques are tested and verified by quantitative analysis of the emerging jet to identify the influence of the surrounding fluid on the HPWJ.

The HPWJ process known from industrial applications can be adapted to the field of rock drilling. In this specific case, the HPWJ is used to cut and destroy rock in deep geothermal reservoirs. The process is known as jet drilling. Although there have been research activities in this field, the process itself is not well understood so far and practical applications are rare. Therefore, the aim of our work is the visualisation of the process to increase the knowledge of waterjet and rock interactions.

High speed photography in terms of shadowgraph experiments is used for visualisation. Moreover, an estimation of the fluid velocity on the boundary of the HPWJ in air is performed. For this, the shadowgraph images are evaluated with the double-frame technique well known with particle image velocimetry (PIV). Analysis of both the structure and the velocity distribution of the HPWJ in water is done by combined PIV and laser induced fluorescence (LIF) analysis with fluorescent dye.
\end{abstract}

\section{Keywords}

PIV, LIF, velocity distribution, jet flow, spray analysis

\section{Introduction}

The use of geothermal energy for heat and power exploitation as a renewable energy resource can contribute to a more sustainable energy supply. Enhanced geothermal systems (EGS) enable the recovery of geothermal energy even in surroundings that do not have a naturally hydrothermal system [1,2]. Based on an injection well drilled into hard crystalline rock in up to $3000 \mathrm{~m}$ depth, several laterals are drilled into the rock formation to create an EGS.

The creation of these laterals can be realized by the use of a HPWJ, a process known as radial jet drilling. Jet drilling provides major advantages over conventional mechanical drilling techniques, namely higher drilling speed, less tool wear and reduced environmental risk. However, jet drilling shows drawbacks when drilling into hard crystalline rocks, which represents a challenge to the utilization for EGS. To overcome this drawback, the jetting process has to be optimized towards the implementation for hard crystalline rock drilling. Although there has been research on jet drilling since the 1960s, the process of water jetting of rocks itself is barely understood in terms of physics and fluid mechanics [3-5].

Therefore, the objective of our research project is to enhance this process knowledge with a focus on the interaction between the HPWJ and the rock surface under realistic conditions. The interaction of the HPWJ and the rock surface can be accessed by determination of the jet energy impact on the rock surface. The energy impact is characterized by geometrical information about the jet as well as the corresponding velocity distribution. For the purpose of quantitative analysis of the geometrical information, the HPWJ can be considered to be a highly dense spray. Thus, relevant spray parameters can be evaluated with common spray analysis techniques to quantify the different influences on the formation of the HPWJ. PIV analysis and the appendant correlation algorithm give information about velocities of the HPWJ. Combining these data, the energy impact of the HPWJ for different operating parameters on the rock surface can be estimated. The determination of the energy impact also contributes to the understanding of the rock destroying mechanism of a HPWJ in different stand-off distances to the rock surface. 
In this work, visualisation and optical measurement techniques are chosen to investigate the process. As there is a lack of optical access in a genuine bore hole in high depth, this visualisation is done gradually starting with the development of a suitable optical measurement technique and progresses towards more realistic designs and conditions. The optical measurement technique is further developed for the analysis of a highly dense, high Reynolds number (currently up to $12.3 \times 10^{4}$ ) jet flow in different fluids. For this purpose, two different methods are tested and successfully applied to the jet flow:

- The application of the shadow method with double-pulsed, non-coherent back illumination in combination with PIV evaluation based on a cross-correlation algorithm and

- A combined PIV and LIF technique with double-pulsed laser light sheet illumination under a $90^{\circ}$ angle relative to the observation direction.

The potential application is verified by a parameter study of the operation parameter's influence as well as the surrounding fluid's influence on the formation of the HPWJ.

The visualisation of a flat nozzle jet at high Reynolds numbers has been performed under atmospheric conditions in air by Zelenak et al. [6]. They demonstrated the applicability of the shadow method with combined PIV correlation algorithm on this less dense, high Reynolds number jet to visualise the jet structure and analyse velocity fields. However, this method shows disadvantages in the near-nozzle region where the jet has a high density. For high Reynolds number water jets, visualisation has been frequently done by shadow method (e.g. $[7,8])$. In addition to that, analysis of the velocity fields of both low and high Reynolds number water jets is commonly done by PIV (e.g. $[9,10]$ ). By combining both the PIV and LIF technique, it is possible to access the jet structure as well as velocity fields at the same time. Fukushima et al. [11] and Webster et al. [12] applied this combination to turbulent jets with Reynolds numbers of 2,000 and 3,000, respectively, with one camera for PIV and one camera for LIF, respectively.

For our research aim, a suitable measurement technique for high Reynolds number, highly dense water jets in a limited space and in different fluids is required. Concerning this, possible limitations are the high density of the jet for the shadow analysis in air, whereas the combination of the high Reynolds number and the small spatial expansions are possible limitations to the PIV and LIF analysis in water. Therefore, the shadow method with PIV correlation algorithm for the jet in air and the combined PIV and LIF technique with one camera for the jet in water are tested and verified to obtain simultaneous information about the jet structure and the velocity.

\section{Experimental setup}

In this contribution, the applicability of established optical measurement techniques for investigation of the HPWJ cutting process are tested and validated. Challenges to the application of these optical measurement techniques are the high velocities, the very limited spatial expansions and the dense liquid jet. Therefore, experiments with a broad parameter variety are performed to establish a reliable and reproducible measurement technique. A successful establishment of the measurement technique can be used for further experiments concerning the interaction between the HPWJ, the surrounding fluid and the rock surface.

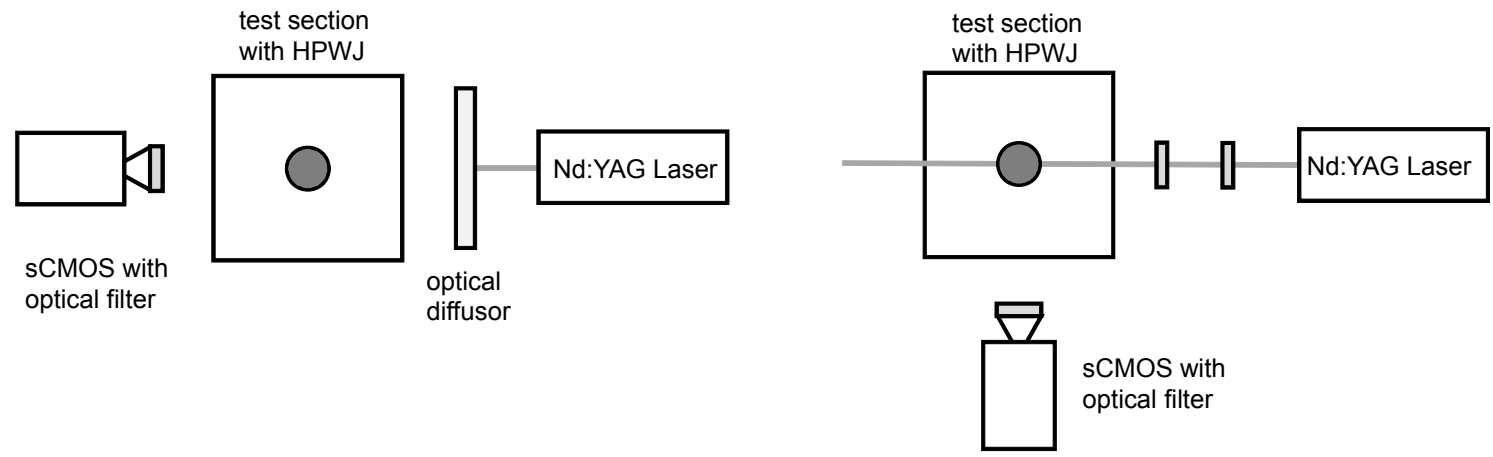

Figure 1. Schematic view of shadowgraph setup for experiments in air (left) and the PIV setup for experiments in water (right) from the top

A high pressure pump (delivering up to $15 \mathrm{dm}^{3} \mathrm{~min}^{-1}$ at a maximum operating pressure of $15 \mathrm{MPa}$ ) and a pressure atomizer nozzle are used to generate the water jet. Experiments are performed for three different nozzle diameters $d_{N}(1.8 \mathrm{~mm}, 2.0 \mathrm{~mm}, 2.3 \mathrm{~mm})$, allowing a parameter range for the nozzle output pressure $p_{N}$ of up to 
$10 \mathrm{MPa}$ and maximum flow rates $\mathrm{Q}_{\mathrm{N}}$ of up to $14 \mathrm{dm}^{3} \mathrm{~min}^{-1}$. The flow rate is measured by electromagnetic inductive flow metering (Optiflux 1300, Krohne). The output pressure is determined by a pressure sensor (BSP00JP, Balluff) $30 \mathrm{~mm}$ upstream of the nozzle. For the two different experiments, the HPWJ is introduced in air or water, respectively. A schematic assembly of the experimental set up for both the shadowgraph system and the PIV system is illustrated in figure 1. The shadowgraph setup is used for experiments in air, whereas the PIV setup is used for experiments in water. A summary of the experimental conditions is given in table 1.

Table 1. Summary of experimental conditions.

\begin{tabular}{ll|c|c}
\hline \hline \multicolumn{2}{c|}{ Parameter } & Unit & Value \\
\hline \hline nozzle diameter & $\mathrm{d}_{\mathrm{N}}$ & $\mathrm{mm}$ & $1.8,2.0,2.3$ \\
nozzle pressure & $\mathrm{p}_{\mathrm{N}}$ & $\mathrm{MPa}$ & $0.1-10$ \\
flow rate & $\mathrm{Q}$ & $\mathrm{dm}^{3} \mathrm{~min}^{-1}$ & $2.0-14.0$ \\
jet velocity & $\mathrm{u}_{\mathrm{J}}$ & $\mathrm{m} \mathrm{s}^{-1}$ & $8.0-130.0$ \\
Reynolds number & $\mathrm{Re}_{\mathrm{N}}$ & - & $1.2 \times 10^{4}-12.3 \times 10^{4}$
\end{tabular}

\section{Visualisation and velocity measurements in air}

High speed photography with a shadowgraph system is used for visualisation of the HPWJ in air and estimation of the velocity distribution. Due to the high velocities of up to $130 \mathrm{~m} \mathrm{~s}^{-1}$ and the small size of the field of view $(20 \mathrm{~mm} \times 20 \mathrm{~mm})$, very short light pulses are needed to capture images of the flow structures without motion blur. Therefore, a shadowgraph system with a light pulse of $20 \mathrm{~ns}$ is selected. This shadowgraph system consists of a 16 bit CMOS camera (sCMOS, LaVision) with a Zeiss Yashica macro lens and a Nd:YAG double-pulse laser (repetition rate $15 \mathrm{~Hz}$, wavelength $532 \mathrm{~nm}$, pulse length $5 \mathrm{~ns}$, maximum energy output per pulse 200 mJ, Quantel Evergreen200) and a laser guiding arm with an optical diffuser, transforming the laser pulse in 20 ns non-coherent pulse. The laser is equipped with a Metrolux laser attenuator that allows stable laser operation at high laser output power and use of an optical diffuser with a maximum laser power input of $50 \mathrm{~mJ}$. The optical diffuser itself consists of laser beam shaping optics, a fluorescently labelled plastic plate and a diffuse screen. The $5 \mathrm{~ns}$ laser light pulse enters the diffuser, is widened by the optics and excites the florescence on the plastic plate positioned under a $45^{\circ}$ angle. A frequency-shifted, non-coherent $20 \mathrm{~ns}$ light pulse is emitted from the plastic plate and the light is guided to the diffuse screen. Applying this setup, it is possible to acquire images with a motion blur of $1-$ 2 pixels under the given experimental conditions. The camera and the laser are synchronized by a timing unit. Using the double-frame mode of the camera and the dual pulse laser, it is also possible to evaluate velocity information from the shadowgraph images. This is especially applied for the HPWJ in air as the reflecting phase boundary makes PIV measurements difficult.

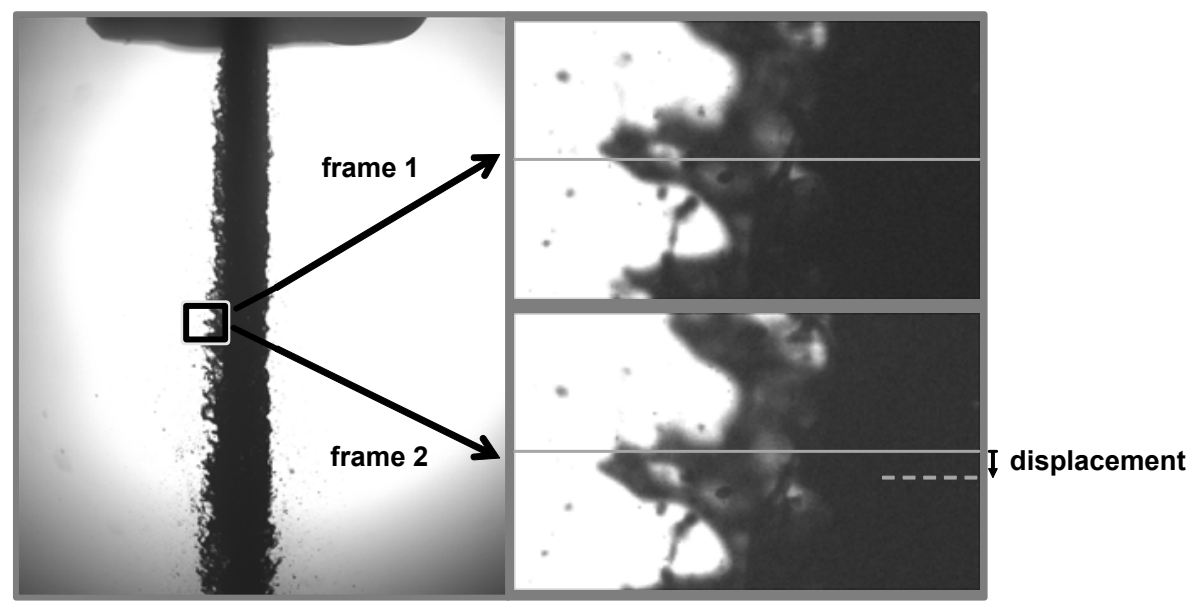

Figure 2. Illustration of the cross-correlation evaluation on the interface structures of the HPWJ in air $\left(\mathrm{d}_{\mathrm{N}}=1.8 \mathrm{~mm}, \mathrm{p}_{\mathrm{N}}=6 \mathrm{MPa}\right.$, $\left.\mathrm{Q}=11 \mathrm{dm}^{3} \mathrm{~min}^{-1}, \operatorname{Re}_{\mathrm{N}}=9.9 \times 10^{4}\right)$. The detailed images on the right show one frame of the double-frame image with dimensions of $1 \times 2 \mathrm{~mm}$, respectively, also including a reference line and the resulting displacement. The structures show a pixel shift of 10 pixels in average from frame 1 to frame 2 .

For every shadow experiment a series of 1000 double-frame images is taken. For spray parameter analysis, image pre-processing is needed. In a first step, the images are inverted, afterwards an appropriate intensity threshold for the determination of the jet boundary is set. The set of 1000 double-frame images are analysed and the mean value of each spray parameter for a single set is taken. Evaluation of the velocity information from the shadowgraph images is done by correlation on interface structures on the boundary of the HPWJ. Therefore, the 
double-frame images are masked before correlation. The principle of correlation on interface structures is illustrated in figure 2.

\section{Visualisation and velocity measurements in water}

In addition to the shadowgraph images, combined PIV and LIF analysis is performed for visualisation of the HPWJ in water and measurement of the velocity distribution. Due to the high density of the spray, fluorescent tracers are used to obtain information of the velocity distribution in the HPWJ. The camera is equipped with a high pass filter with a cut-on at $540 \mathrm{~nm}$ to ensure that no undesired reflections from the laser light but only the light emitted by the fluorescent dye is recorded. Water soluble vinyl paint with rhodamine B (emission peak at $565 \mathrm{~nm}$ ) as fluorophore is used both as fluorescent dye and tracer, as the vinyl paint does also contain fluorescent, solid particles. The use of this fluorescent paint permits simultaneous PIV and LIF analysis. The evaluation of this simultaneous measurement technique has been introduced by Lindken and Merzkirch [13] and has also been successfully adapted to other applications $[14,15]$. The technique is based on the separation of different grey level values. In this work, the grey levels are obtained from the background, the image of the dissolved dye and the fluorescent particles in increasing order, illustrated in figure 3. Consequently, simultaneous measurement of the HPWJ interface as well as velocity distribution can be applied by appropriate thresholding.

Similar to the shadow experiments, a series of 1000 double-frame images is also taken for every measurement point. PIV analysis is done by cross-correlation of the double-frame images. To obtain the shape of the HPWJ, image post-processing with an appropriate threshold is needed. Subsequently, the images can also be used to analyse spray parameters.
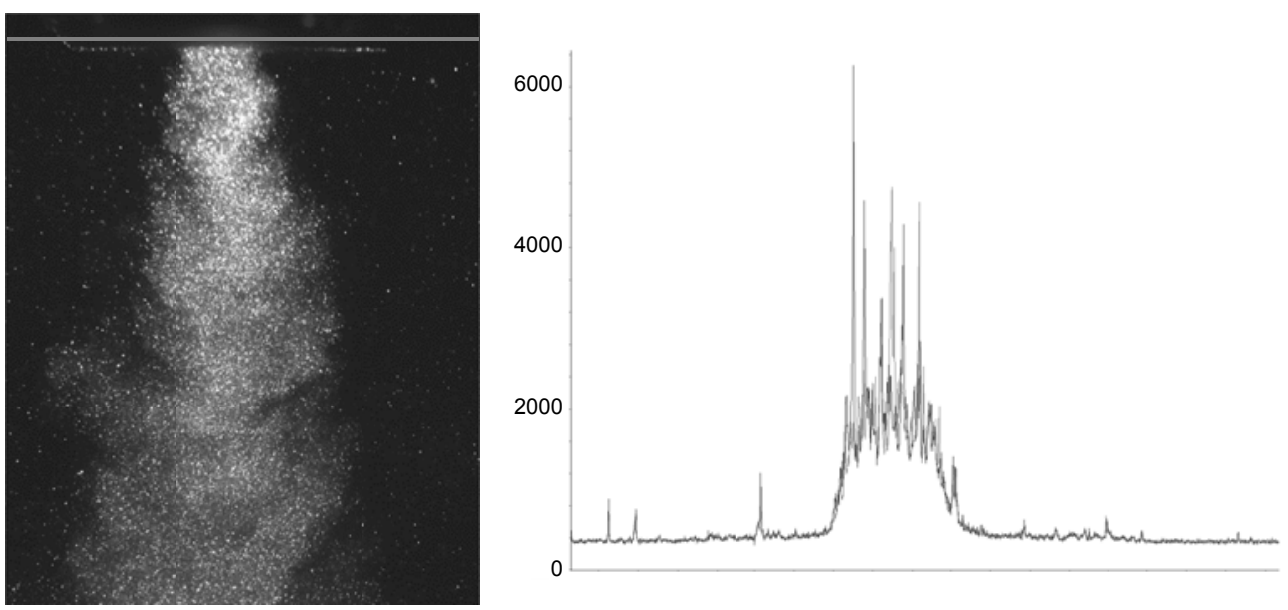

Figure 3. Example of a combined instantaneous PIV and LIF image with different grey levels of the HPWJ in water $\left(\mathrm{d}_{\mathrm{N}}=1.8 \mathrm{~mm}, \mathrm{p}_{\mathrm{N}}=0.1 \mathrm{MPa}, \mathrm{Q}=2.3 \mathrm{dm}^{3} \mathrm{~min}^{-1}, \mathrm{Re}_{\mathrm{N}}=1.5 \times 10^{4}\right)$. On the right, the grey value distribution along the grey line in the left image is shown. The fluorescent dye seeded jet creates grey values about 1500 counts, while the unseeded surrounding water is at 300 counts. Fluorescent tracer particles appear as peaks at around 4000 counts.

\section{Velocity distribution of the HPWJ}

Applying the cross-correlation algorithm on the interface structures of the HPWJ in air, the velocity distribution at the boundary of the HPWJ can be determined. To prevent uncertainties in the correlation algorithm, the core of the HPWJ is masked out [6]. For verification of the correlation results, the jet velocity $u_{J}$ can be estimated by the continuity equation, taking into account the measured flow rate $Q$ and the physical nozzle cross-sectional area $A_{N}$. Figure 4 contrasts the obtained velocity values. Differently than expected, the velocity distribution on the boundary of the HPWJ by correlation of the shadow images is higher than the one obtained by the above mentioned calculation. This discrepancy is due to the assumption of single phase, liquid flow. In air, the visualisation does not show the existence of a second, gaseous phase. That is why the first velocity estimation is done with the assumption of single phase, liquid flow. In contrast to that, visualisation in water shows the formation of gas bubbles right at the nozzle outlet. 

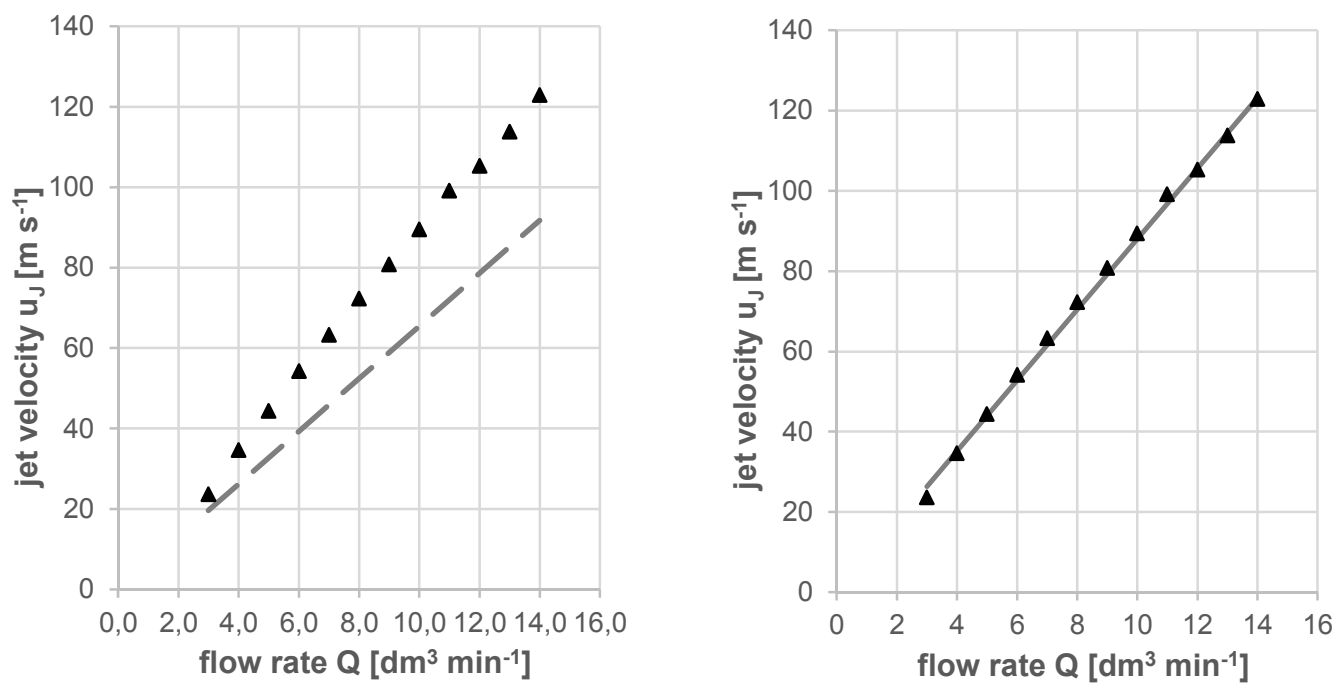

Figure 4. Velocity at the interface of the HPWJ in air, $d_{N}=1.8 \mathrm{~mm}$. Values determined by eq. (1) with assumption of single phase flow (left, grey dashed line) and two phase flow (right, grey line). Values determined by PIV cross-correlation are given in both diagrams (black triangles).

Possible reasons for the formation of a gaseous phase are already dissolved gas in the water or the occurrence of cavitation, which, in addition, can be favoured by outgassing of the dissolved gas. The existence of a gaseous phase can block a part of the nozzle outlet cross-sectional area. This blocking reduces the effective outlet crosssectional area $A_{\text {eff, }}$ resulting in higher outlet velocities [15]. Therefore, the correlated velocities are higher due to a smaller effective outlet cross-sectional area in comparison to the physical cross-sectional area used for velocity estimation.

Due to the occurrence of the gaseous phase, the application of the combined PIV and LIF analysis for the HPWJ in water is limited. The gas bubbles cause scattering both of the laser light and the light emitted by the fluorescent dye. This is a major drawback because an analysis of the velocity distribution in the core of the jet close to the nozzle outlet is not feasible with this experimental setup. Nevertheless, from a distance of about 6-7 nozzle diameters downwards, no gaseous phase is present and a correlation of the double-frame images is possible. Thus, comparing the velocity at the boundary of the HPWJ both in air and water is plausible in this region. As expected, the correlation results indicate that the velocity of the HPWJ in water is smaller than in air [e.g. 17].

\section{Estimation of the gas proportion from velocity data}

Due to the presence of a gaseous phase, the effective nozzle outlet cross-sectional area is reduced. However, the resulting effective nozzle outlet cross-sectional area $A_{\text {eff }}$ can be determined by the correlated velocities in air $\mathrm{u}_{\mathrm{J}^{*}}$ and the measured flow rate $\mathrm{Q}$ in the nozzle feeding pipe before cavitation occurs, eq. (1).

$$
A_{\text {eff }}=Q / u_{J^{*}}
$$

This leads to an effective outlet cross-sectional area which is up to one fourth smaller than the physical outlet cross-section. According to the relationship between the effective outlet cross-sectional area $A_{\text {eff }}$ and the gas proportion $\mathrm{x}$, the latter can be estimated at the nozzle outlet, eq. (2).

$$
x=\left(A_{N}-A_{\text {eff }}\right) / A_{\text {eff }}
$$

Consequently, the void fraction can be quantified to be $20-26 \%$, increasing with decreasing physical nozzle outlet cross-sectional area or diameter. For example, the estimated velocity values by continuity equation with a void fraction of $26 \%$ for the smallest nozzle outlet diameter $d_{N}=1.8 \mathrm{~mm}$ are shown in figure 4 . Although the gas bubbles are a limitation to the optical access of the near nozzle jet, the existence of those gas bubbles can be favourable in the rock destroying mechanism [18].

\section{Structure of the HPWJ}

The pressure atomizer nozzles used for the experiments are designed for applications in the open air. Under these conditions, they have a very small spray angle to generate a full jet with a high energy impact. The spray 
cone angle of the HPWJ is a relevant spray parameter to analyse the influence of the surrounding fluid. An increase in the spray angle indicates an increase in the interaction of the HPWJ and the surrounding fluid.

For the HPWJ in air, the spray angle for all examined nozzles is smaller than $4^{\circ}$, showing a moderate increase with increasing flow rate, pressure and nozzle outlet diameter, respectively. In contrast to that but similar to literature, the HPWJ in water shows a significantly wider spray angle at the same experimental conditions. The increase in the spray angle is caused by higher shear forces at the jet interface [e.g. 17,19]. As an example, figure 5 shows the comparison of the time averaged HPWJ structure in air and water respectively.

In addition to that, the structure on the boundary of the HPWJ changes from a smooth to a rougher interface, showing clearly visible interface structures. Comparing instantaneous images, it is also visible that, in the field of view, the HPWJ does not break up in air, whereas in water the HPWJ does break up. This is also in accordance with literature [e.g. 17,19]. Two examples of instantaneous images are shown in figure 6 . The break up in water is due to the higher viscosity of water in contrast to air. The resulting shear forces are higher, causing the HPWJ to break up or atomize.
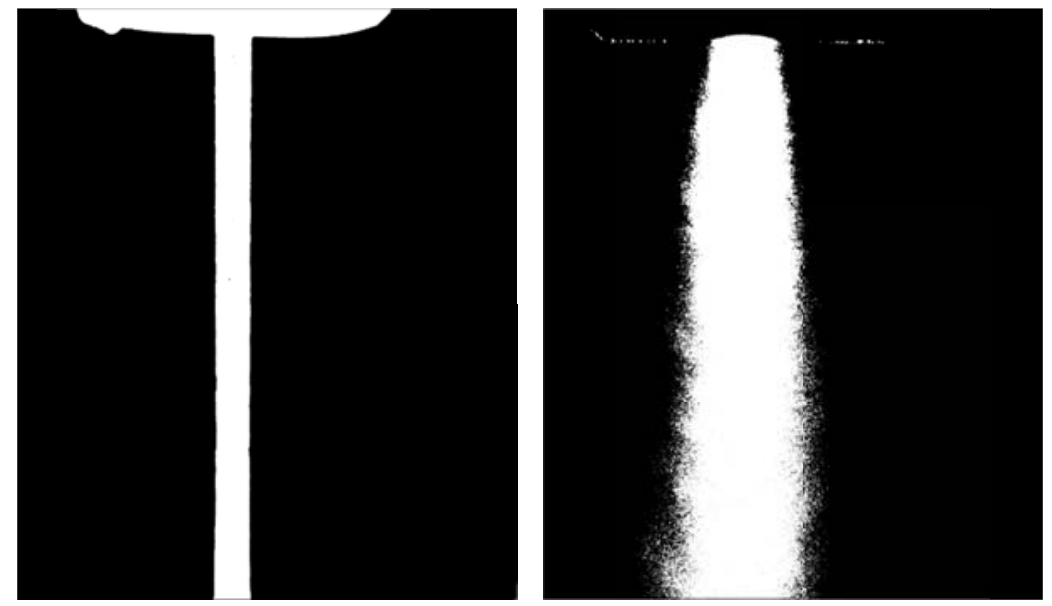

Figure 5. Comparison of the time averaged structure of the HPWJ in air (left) and water (right), $d_{N}=1.8 \mathrm{~mm}, \mathrm{p}_{\mathrm{N}}=0.1 \mathrm{MPa}$, $\mathrm{Q}=2.3 \mathrm{dm}^{3} \mathrm{~min}^{-1}, \operatorname{Re}_{\mathrm{N}}=1.5 \times 10^{4}$
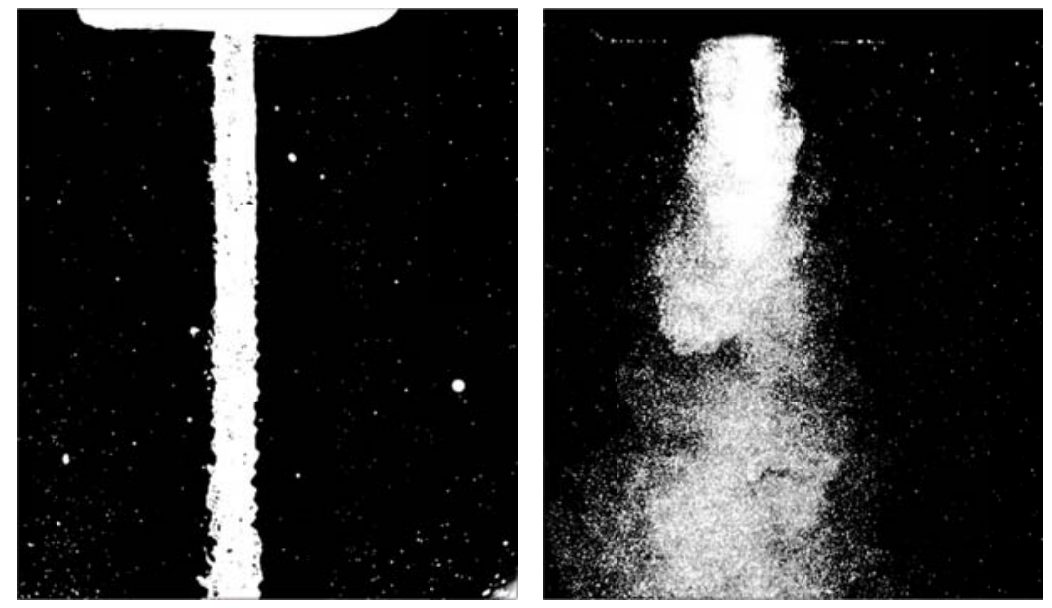

Figure 6. Comparison of the instantaneous structure of the HPWJ in air (left) and water (right), $\mathrm{d}_{\mathrm{N}}=1.8 \mathrm{~mm}, \mathrm{p}_{\mathrm{N}}=0.1 \mathrm{MPa}$, $Q=2.3 \mathrm{dm}^{3} \min ^{-1}, \operatorname{Re}_{\mathrm{N}}=1.5 \times 10^{4}$

\section{Conclusions}

Both high speed photography in terms of shadowgraph images and the combined PIV and LIF technique are successfully adopted to the present experimental setup and conditions. Both techniques can be applied to simultaneously visualise and analyse the HPWJ with high Reynolds numbers of currently up to $12.3 \times 10^{4}$ for drilling applications. To ensure the applicability to the given circumstances, further experiments with absence of a gaseous phase will be performed. Nevertheless, the experiments presented in this contribution give first hints about the influence of the surrounding fluid on a HPWJ which are in accordance with literature data. 
The here proven measurement techniques can be used to obtain a model for the energy impact on the rock surface. Both the structural and velocity information indicate that the energy impact under realistic borehole conditions, which means in a fluid other than air, is less. Therefore, it is key to expand the knowledge of the generation and development of the HPWJ in different fluids to determine the optimal set up for rock destruction and a successful technology transfer of the HPWJ process to drilling applications. To achieve this objective, the proven experimental techniques for water can also be applied for measurements in slurry and drilling mud in future work.

\section{Acknowledgements}

This work is being funded through the "FH-Struktur2016" venue for universities of applied sciences by the ministry for innovation, science and research of the state of Nordrhein-Westfalen, Germany (AZ: 322-8.03.04.02).

\section{Nomenclature}

$A_{\text {eff }} \quad$ effective nozzle cross-sectional area [mm]

$\mathrm{A}_{\mathrm{N}} \quad$ nozzle cross-sectional area [mm]

$\mathrm{d}_{\mathrm{N}} \quad$ nozzle diameter $[\mathrm{mm}]$

$\dot{m} \quad$ mass flow $\left[\mathrm{kg} \mathrm{s}^{-1}\right]$

$\mathrm{p}_{\mathrm{N}} \quad$ nozzle pressure [MPa]

$Q \quad$ flow rate $\left[\mathrm{dm}^{3} \mathrm{~min}^{-1}\right]$

$\mathrm{Re}_{\mathrm{N}} \quad$ Reynolds number at nozzle exit [-]

$\mathrm{u}_{\mathrm{J}} \quad$ jet velocity $\left[\mathrm{m} \mathrm{s}^{-1}\right.$ ]

$\mathrm{u}_{\mathrm{J}^{*}} \quad$ correlated jet velocity $\left[\mathrm{m} \mathrm{s}^{-1}\right]$

$\rho_{\mathrm{L}} \quad$ liquid density $\left[\mathrm{kg} \mathrm{m}^{-3}\right]$

\section{References}

[1] Tester, J.W., et al., 2006, "The Future of Geothermal Energy - Impact of Enhanced Geothermal Systems (EGS) on the United States in the 21st Century".

[2] Finsterle, S., Zhang, Y., Pan, L., Dobson, P, Oglesby, K., 2013, Geothermics, 47, pp. 104-115.

[3] Farmer, I. and Attewell, P., 1965, International Journal of Rock Mechanics and Mining Sciences \& Geomechanics Abstracts, 2 (2), pp. 135 - 153.

[4] Brook, N., Summers, D. A., 1969, International Journal of Rock Mechanics and Mining Sciences \& Geomechanics Abstracts, 6 (3), pp. 249-258.

[5] Hood, M., Nordlund, R., Thimons, E., 1990, International Journal of Rock Mechanics and Mining Sciences \& Geomechanics Abstracts, 27 (2), pp. 77-86.

[6] Zelenak, M., Foldyna, J., Scucka, J., Hloch, S., Riha, Z., 2015, Measurement, 72, pp. 1-8.

[7] Donald, M.B., Singer, H., 1959, Transactions of the Institution of Chemical Engineers, 37, pp. 255-267.

[8] Soyama, H., Yanauchi, Y., Sato, K., Ikohagi, T., Oba, R., Oshima, R., 1996, Experimental Thermal and Fluid Science, 12 (4), pp. 411-416.

[9] Or, C. M., Lam, K. M., Liu, P., 2011, Journal of Hydro-environment Research, 5 (2), pp. 81-91.

[10] Lacagnina, G., Romano, G.P., 2015, Experiments in Fluids, 56 (1), pp. 480-490.

[11] Fukushima C., Aanen L., Westerweel J., Laser Techniques for Fluid Mechanics, pp. 339-356.

[12] Webster, D.R., Roberts, P.J.W., Ra'ad, L., 2001, Experiments in Fluids, 30 (1), pp. 65-72.

[13] Lindken, R., Merzkirch, W., 2002, Experiments in Fluids, 33 (6), pp. 814-825.

[14] Nogueira, S., Soursa, R.G., Pinto, A.M.F.R., Riethmuller, M.L., Campos, J.B.L.M., 2003, Experiments in Fluids, 35 (6), pp. 598-609.

[15] Westerweel, J., Petracci, A., Delfos, R., Hunt, J. C., 2011, Philosophical transactions. Series A, Mathematical, physical, and engineering sciences, 369 (1937), pp. 723-737.

[16] Payri, F., Bermúdez, V., Payri, R., Salvador, F.J., 2004, Fuel, 83 (4-5), pp. 419-431.

[17] Abramomovich, G.N., 1963, "The theory of turbulent jets".

[18] Field, J.E., Camus, J.J., Tinguely, M., Obreschkow, D., Farhat, M., 2012, Wear, 290-291, pp. 154-160.

[19] Hussein, J.H., Capp, S.P., George, W., 1994, Journal of Fluid Mechanics, 258 (1), pp. 31-75. 